\title{
A field expansions method for scattering by periodic multilayered media
}

Alison Malcolm and RKSDavid P. Nicholls and RKS

Citation: The Journal of the Acoustical Society of America 129, 1783 (2011); doi: 10.1121/1.3531931

View online: http://dx.doi.org/10.1121/1.3531931

View Table of Contents: http://asa.scitation.org/toc/jas/129/4

Published by the Acoustical Society of America 


\title{
A field expansions method for scattering by periodic multilayered media
}

\author{
Alison Malcolm \\ Department of Earth, Atmospheric, and Planetary Sciences, Massachusetts Institute of Technology, \\ Cambridge, Massachusetts 02139 \\ David P. Nicholls ${ }^{\text {a) }}$ \\ Department of Mathematics, Statistics, and Computer Science, University of Illinois at Chicago, Chicago, \\ Illinois 60607
}

(Received 7 April 2010; revised 1 December 2010; accepted 1 December 2010)

\begin{abstract}
The interaction of acoustic and electromagnetic waves with periodic structures plays an important role in a wide range of problems of scientific and technological interest. This contribution focuses upon the robust and high-order numerical simulation of a model for the interaction of pressure waves generated within the earth incident upon layers of sediment near the surface. Herein described is a boundary perturbation method for the numerical simulation of scattering returns from irregularly shaped periodic layered media. The method requires only the discretization of the layer interfaces (so that the number of unknowns is an order of magnitude smaller than finite difference and finite element simulations), while it avoids not only the need for specialized quadrature rules but also the dense linear systems characteristic of boundary integral/element methods. The approach is a generalization to multiple layers of Bruno and Reitich's "Method of Field Expansions" for dielectric structures with two layers. By simply considering the entire structure simultaneously, rather than solving in individual layers separately, the full field can be recovered in time proportional to the number of interfaces. As with the original field expansions method, this approach is extremely efficient and spectrally accurate. (C) 2011 Acoustical Society of America. [DOI: 10.1121/1.3531931]
\end{abstract}

PACS number(s): 43.30.Hw, 43.20.El, 43.20.Bi, 43.40.Ph [RKS] Pages: 1783-1793

\section{INTRODUCTION}

The interaction of acoustic and electromagnetic waves with periodic structures plays an important role in a wide range of problems of scientific and technological interest. From grating couplers ${ }^{1-3}$ to nanostructures ${ }^{4}$ to remote sensing, ${ }^{5}$ the ability to simulate in a robust and accurate way the fields generated by such structures is of crucial importance to researchers from many disciplines. In this contribution, we focus upon the robust and high-order numerical simulation of a model for the interaction of pressure waves generated within the earth incident upon layers of sediment near the surface. While we focus on the simplified model of linear acoustic waves in a two-dimensional structure, the core of the algorithm will remain the same for a fully three-dimensional linear elastic simulation (though the implementation details will be significantly more complicated).

This problem is motivated jointly by the recent increased interest in oil exploration in mountainous regions, and the rash of recent large earthquakes, which tend to occur in regions with significant topography. Simulating the seismic wavefield accurately in such regions is key for both imaging (e.g., through waveform inversion, see Virieux and Operto ${ }^{6}$ for a recent review and Bleibinhaus and Rondenay ${ }^{7}$ for a specific discussion of topography in such algorithms) and hazard assessment. $^{8,9}$ A wide array of numerical algorithms have

\footnotetext{
a) Author to whom correspondence should be addressed. Electronic mail: nicholls@math.uic.edu
}

been devised in the past 50 years for the simulation of precisely the problem we consider. The classical finite difference method (FDM) (Refs. 10 and 11), finite element method (FEM) (Refs. 12 and 13), and spectral element method (SEM) (Refs. 14 and 15) are available but suffer from the fact that they discretize the full volume of the model which not only introduces a huge number of degrees of freedom but also raises the difficult question of appropriately specifying a farfield boundary condition explicitly. Furthermore, the FDM, while simple to devise and implement, is not well-suited to the complex geometries of the general layered media. A compelling alternative is surface integral methods ${ }^{16,17}$ (e.g., boundary integral methods-BIMs-or boundary element methods-BEMs) which only require a discretization of the layer interfaces (rather than the whole structure) and which, due to the choice of the Green's function, enforce the far-field boundary condition exactly. While these methods can deliver high-accuracy simulations with greatly reduced operation counts, there are several difficulties which need to be addressed. First, high-order simulations can only be realized with specially designed quadrature rules which respect the singularities in the Green's function (and its derivative, in certain formulations). Additionally, BIM/BEM typically gives rise to dense linear systems to be solved which require carefully designed preconditioned iterative methods (with accelerated matrix-vector products, e.g., by the fast-multipole $\operatorname{method}^{18}$ ) for configurations of engineering interest.

In this work, we describe a boundary perturbation method (BPM) for the numerical simulation of scattering returns from irregularly shaped periodic layered media. We focus upon periodic structures as they arise in a large number 
of engineering applications; however, this choice does simplify our numerical approach (e.g., we may use the discrete Fourier transform to approximate Fourier coefficients). We note that this simplification is also realized for the other methods listed above. Like BIM/BEM, the method requires only the discretization of the layer interfaces (so that the number of unknowns is an order of magnitude smaller than FDM, FEM, and SEM simulations), while it avoids not only the need for specialized quadrature rules but also the dense linear systems characteristic of BIM/BEM. Our approach is a generalization of the "Method of Field Expansions" (FE) described by Bruno and Reitich ${ }^{19-22}$ for dielectric structures with two layers (denoted there the "Method of Variation of Boundaries"). This method is similar in spirit to the "Method of Operator Expansions" (OE) of Milder, ${ }^{23,24}$ Milder and Sharp, 25,26 and Milder" ${ }^{27,28}$ and the "Transformed Field Expansions" (TFE) approach of the Nicholls and Reitich, ${ }^{29-32}$ and these approaches could also be extended in the way we describe here. We save this for future work, however, as the (field expansion) FE approach is the simplest to implement. The FE method was generalized by Hesthaven and collaborators to the case of grating couplers and layered media, ${ }^{1-3}$ precisely the problem we consider here, though we have found their method to be highly inefficient. As we discuss at the end of Sec. III B, their approach relies on the iterative solution of the problem from one layer to the next with the two-layer solver of Bruno and Reitich, ${ }^{20}$ applied sequentially to each pair of layers. After a great number of iterations, this method will eventually converge to the full scattered field at enormous computational cost. We have found that by simply considering the entire structure (more specifically the full set of interfaces), the full field can be recovered simultaneously in time proportional to the number of interfaces. As with the FE method, as it was originally designed by Bruno and Reitich, our new approach is spectrally accurate (i.e., it has convergence rates faster than any polynomial order) due to both the analyticity of the scattered fields with respect to the boundary perturbation and the optimal choice of spatial basis functions which arise naturally from the FE methodology.

The organization of the paper is as follows: In Sec. II, we recall the governing equations of acoustic scattering in a triply layered medium, and in Secs. II A and II B, we describe our FE approach for such media with trivial (flat) and non-trivial (perturbed) layering structure, respectively. In Secs. III, III A, and III B, we repeat these considerations for the general $(M+1)$-layer case. In Sec. IV, we display results of numerical simulations for three- and five-layer structures to demonstrate the accuracy, efficiency, reliability, and flexibility of our new numerical algorithm.

\section{FIELD EXPANSIONS: THREE LAYERS}

For ease of exposition, we begin by describing the case of a triply layered material in two dimensions with nondimensional period $d=2 \pi$. In each of the layers, the (reduced) scattered pressure satisfies the Helmholtz equation with continuity conditions at the upper interface, illumina- tion conditions at the lower interface, and outgoing wave conditions (OWCs) at positive and negative infinity. More precisely, we define the domains

$$
\begin{aligned}
& S_{u}=\{(x, y) \mid y>\bar{g}+g(x)\}, \\
& S_{v}=\{(x, y) \mid \bar{h}+h(x)<y<\bar{g}+g(x)\}, \\
& S_{w}=\{(x, y) \mid y<\bar{h}+h(x)\}
\end{aligned}
$$

with (upward pointing) normals

$$
N_{g}=\left(-\partial_{x} g, 1\right)^{T}, \quad N_{h}=\left(-\partial_{x} h, 1\right)^{T}
$$

and mid-levels $y=\bar{g}, y=\bar{h}$; see Fig. 1 . In each of these domains is a constant-density acoustic medium with velocity $c_{j}(j=u, v, w)$; we assume that plane-wave radiation is incident upon the structure from below:

$$
\tilde{w}(x, y, t)=e^{-i \omega t} e^{i(\alpha x+\beta y)}=e^{-i \omega t} w_{i}(x, y) .
$$

With these specifications, we can define in each layer the parameter $k_{j}=\omega / c_{j}$ which characterizes both the properties of the material and the frequency of radiation in the structure. If the reduced scattered fields (i.e., the full scattered fields with the periodic time dependence factored out) in $S_{u}, S_{v}$, and $S_{w}$ are, respectively, denoted as $\{u, v, w\}=\{u(x, y), v(x, y)$, $w(x, y)\}$, then these functions will be quasiperiodic ${ }^{33}$

$$
\begin{aligned}
& u(x+d, y)=e^{i \alpha d} u(x, y), \quad v(x+d, y)=e^{i \alpha d} v(x, y), \\
& w(x+d, y)=e^{i \alpha d} w(x, y),
\end{aligned}
$$

and the system of partial differential equations to be solved is

$$
\begin{aligned}
& \Delta u+k_{u}^{2} u=0, \quad y>\bar{g}+g(x), \\
& \mathcal{B}\{u\}=0, \quad y \rightarrow \infty
\end{aligned}
$$

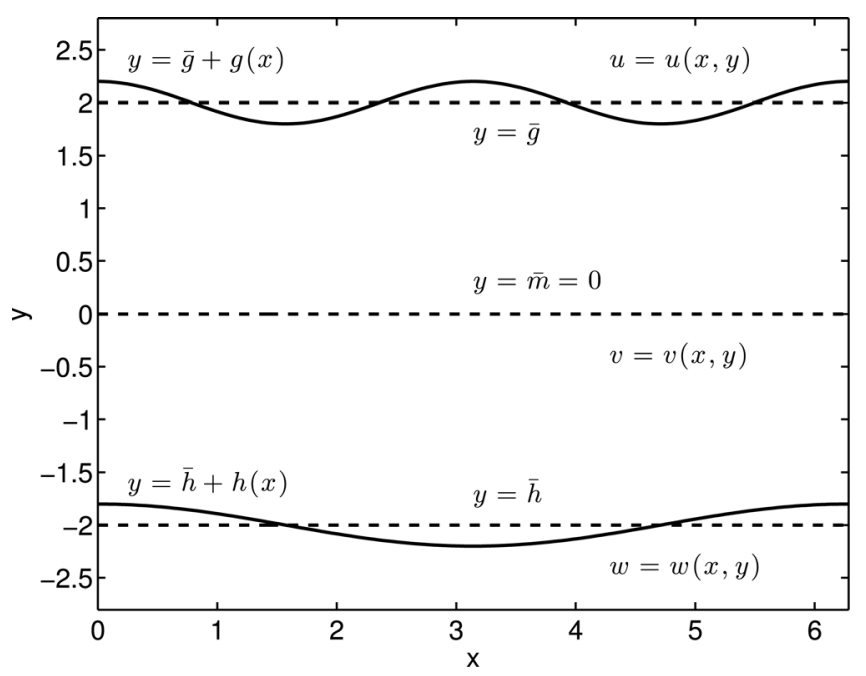

FIG. 1. Problem configuration with layer boundaries in solid lines and midlevels in dashed lines. Here $\bar{g}=2, \bar{h}=-2, g(x)=0.2 \cos (x), h(x)=0.2$ $\cos (2 x)$, and $\bar{m}=0$. 


$$
\begin{aligned}
& \Delta v+k_{v}^{2} v=0, \quad \bar{h}+h(x)<y<\bar{g}+g(x), \\
& u-v=0, \quad \partial_{N_{g}}(u-v)=0, \quad y=\bar{g}+g(x), \\
& \Delta w+k_{w}^{2} w=0, \quad y<\bar{h}+h(x), \\
& \mathcal{B}\{w\}=0, \quad y \rightarrow-\infty, \\
& v-w=\xi, \quad \partial_{N_{h}}(v-w)=\psi, \quad y=\bar{h}+h(x),
\end{aligned}
$$

where

$$
\begin{aligned}
& \xi(x)=-w_{i}(x, \bar{h}+h(x)), \\
& \psi(x)=-\left[\partial_{N_{h}} w_{i}(x, y)\right]_{y=\bar{h}+h(x)} .
\end{aligned}
$$

In these equations, the operator $\mathcal{B}$ enforces the condition that scattered solutions must either be "outgoing" (upward in $S_{u}$ and downward in $S_{w}$ ) if they are propagating or "decaying" if they are evanescent. We make this "Outgoing Wave Condition" (Ref. 33) more precise in the Fourier series expression for the exact solution, see Eq. (3) below.

The quasiperiodic solutions of the Helmholtz equations - (2a), (2c), and (2e)—and the OWCs—-(2b) and (2f) are given by ${ }^{33}$

$$
\begin{aligned}
u(x, y)= & \sum_{p=-\infty}^{\infty} a_{p} \exp \left(i\left(\alpha_{p} x+\beta_{u, p}(y-\bar{g})\right)\right), \\
v(x, y)= & \sum_{p=-\infty}^{\infty} b_{p} \exp \left(i\left(\alpha_{p} x-\beta_{v, p}(y-\bar{m})\right)\right) \\
& +\sum_{p=-\infty}^{\infty} c_{p} \exp \left(i\left(\alpha_{p} x+\beta_{v, p}(y-\bar{m})\right)\right), \\
w(x, y)= & \sum_{p=-\infty}^{\infty} d_{p} \exp \left(i\left(\alpha_{p} x-\beta_{w, p}(y-\bar{h})\right)\right),
\end{aligned}
$$

where $\bar{m}=(\bar{g}+\bar{h}) / 2$, and the OWC mandates that we choose the positive sign in front of $\beta_{u, p}$ in Eq. (3a) and the negative sign in front of $\beta_{w, p}$ in Eq. (3c). These formulas are valid provided that $(x, y)$ are outside the grooves, i.e.,

$$
\begin{aligned}
(x, y) \in & \left\{y>\bar{g}+|g|_{L^{\infty}}\right\} \cup\left\{\bar{h}+|h|_{L^{\infty}}<y<\bar{g}-|g|_{L^{\infty}}\right\} \\
& \cup\left\{y<\bar{h}-|h|_{L^{\infty}}\right\} .
\end{aligned}
$$

In these equations

$$
\alpha_{p}=\alpha+(2 \pi / d) p, \quad \beta_{j, p}= \begin{cases}\sqrt{k_{j}^{2}-\alpha_{p}^{2}} & \alpha_{p}^{2}<k_{j}^{2} \\ i \sqrt{\alpha_{p}^{2}-k_{j}^{2}} & \alpha_{p}^{2}>k_{j}^{2}\end{cases}
$$

$j=u, v, w$, and $d$ is the period of the structure. Again, the OWC determines the choice of sign for $\beta_{j, p}$ in the evanescent case $\alpha_{p}^{2}>k_{j}^{2}$. The boundary conditions-(2d) and $(2 \mathrm{~g})-$ determine the coefficients $\left\{a_{p}, b_{p}, c_{p}, d_{p}\right\}$.

\section{A. Trivial interfaces}

In the case, where the interfaces are flat (i.e., $g=h \equiv 0$ ) then the equations for $\vec{z}_{p}=\left(a_{p}, b_{p}, c_{p}, d_{p}\right)^{T}$ become quite straightforward. Equations (3), (2d), and ( $2 \mathrm{~g})$ mandate that

$$
\begin{aligned}
0= & \sum_{p=-\infty}^{\infty} \exp \left(i \alpha_{p} x\right)\left\{a_{p}-b_{p} \exp \left(-i \beta_{v, p}(\bar{g}-\bar{m})\right)\right. \\
& \left.-c_{p} \exp \left(i \beta_{v, p}(\bar{g}-\bar{m})\right)\right\} \\
0= & \sum_{p=-\infty}^{\infty} \exp \left(i \alpha_{p} x\right)\left\{\left(i \beta_{u, p}\right) a_{p}-\left(-i \beta_{v, p}\right) b_{p}\right. \\
& \times \exp \left(-i \beta_{v, p}(\bar{g}-\bar{m})\right)-\left(i \beta_{v, p}\right) c_{p} \\
& \left.\times \exp \left(i \beta_{v, p}(\bar{g}-\bar{m})\right)\right\} \\
\xi(x)= & \sum_{p=-\infty}^{\infty} \exp \left(i \alpha_{p} x\right)\left\{b_{p} \exp \left(-i \beta_{v, p}(\bar{h}-\bar{m})\right)\right. \\
& \left.+c_{p} \exp \left(i \beta_{v, p}(\bar{h}-\bar{m})\right)-d_{p}\right\} \\
\psi(x)= & \sum_{p=-\infty}^{\infty} \exp \left(i \alpha_{p} x\right)\left\{\left(-i \beta_{v, p}\right) b_{p}\right. \\
& \times \exp \left(-i \beta_{v, p}(\bar{h}-\bar{m})\right)+\left(i \beta_{v, p}\right) c_{p} \\
& \left.\times \exp \left(i \beta_{v, p}(\bar{h}-\bar{m})\right)-\left(-i \beta_{w, p}\right) d_{p}\right\} .
\end{aligned}
$$

Upon expansion of $\xi(x)$ and $\psi(x)$ in Fourier series

$$
\xi(x)=\sum_{p=-\infty}^{\infty} \hat{\xi}_{p} \exp \left(i \alpha_{p} x\right), \quad \psi(x)=\sum_{p=-\infty}^{\infty} \hat{\psi}_{p} \exp \left(i \alpha_{p} x\right),
$$

we can write Eq. (5) "wavenumber-by-wavenumber" as

$$
\mathcal{A}_{p} \vec{z}_{p}=\vec{r}_{p}
$$

where

$$
\mathcal{A}_{p}=\left(\begin{array}{cccc}
1 & -\exp \left(-i \beta_{v, p}(\bar{g}-\bar{m})\right) & -\exp \left(i \beta_{v, p}(\bar{g}-\bar{m})\right) & 0 \\
\left(i \beta_{u, p}\right) & \left(i \beta_{v, p}\right) \exp \left(-i \beta_{v, p}(\bar{g}-\bar{m})\right) & -\left(i \beta_{v, p}\right) \exp \left(i \beta_{v, p}(\bar{g}-\bar{m})\right) & 0 \\
0 & \exp \left(-i \beta_{v, p}(\bar{h}-\bar{m})\right) & \exp \left(i \beta_{v, p}(\bar{h}-\bar{m})\right) & -1 \\
0 & -\left(i \beta_{v, p}\right) \exp \left(-i \beta_{v, p}(\bar{h}-\bar{m})\right) & \left(i \beta_{v, p}\right) \exp \left(i \beta_{v, p}(\bar{h}-\bar{m})\right) & \left(i \beta_{w, p}\right)
\end{array}\right)
$$

and

$$
\vec{r}_{p}=\left(0,0, \hat{\xi}_{p}, \hat{\psi}_{p}\right)^{T}
$$

While not exactly the same, this algorithm (with trivial interfaces) is very much in the spirit of the "Reflectivity Method.",34 


\section{B. Non-trivial interfaces}

To deal with non-trivial interfaces, we once again appeal to the representations (3) which satisfy the Helmholtz equations and OWCs. As before, the boundary conditions (2d) and (2g) determine the coefficients $\vec{z}_{p}=\left(a_{p}, b_{p}, c_{p}\right.$, $\left.d_{p}\right)^{T}$, however, these conditions must be understood as $g$ and $h$-dependent equations.

The FE method (Ref. 20) as applied to Eq. (5) supposes that if the interfaces are small perturbations of the flat interface case, $g(x)=\varepsilon f(x)$ and $h(x)=\varepsilon s(x)$, then the fields $\{u, v, w\}=\{u(x, y ; \varepsilon), v(x, y ; \varepsilon), w(x, y ; \varepsilon)\}$ will depend analytically upon $\varepsilon$, allowing the Taylor expansion about $\varepsilon=0$

$$
\begin{aligned}
u(x, y ; \varepsilon)= & \sum_{p=-\infty}^{\infty} a_{p}(\varepsilon) \exp \left(i\left(\alpha_{p} x+\beta_{u, p}(y-\bar{g})\right)\right) \\
= & \sum_{p=-\infty}^{\infty} \sum_{n=0}^{\infty} a_{p, n} \varepsilon^{n} \exp \left(i\left(\alpha_{p} x+\beta_{u, p}(y-\bar{g})\right)\right) \\
v(x, y ; \varepsilon)= & \sum_{p=-\infty}^{\infty} b_{p}(\varepsilon) \exp \left(i\left(\alpha_{p} x-\beta_{v, p}(y-\bar{m})\right)\right) \\
& +\sum_{p=-\infty}^{\infty} c_{p}(\varepsilon) \exp \left(i\left(\alpha_{p} x+\beta_{v, p}(y-\bar{m})\right)\right) \\
= & \sum_{p=-\infty}^{\infty} \sum_{n=0}^{\infty} b_{p, n} \varepsilon^{n} \exp \left(i\left(\alpha_{p} x-\beta_{v, p}(y-\bar{m})\right)\right) \\
& +\sum_{p=-\infty}^{\infty} \sum_{n=0}^{\infty} c_{p, n} \varepsilon^{n} \exp \left(i\left(\alpha_{p} x+\beta_{v, p}(y-\bar{m})\right),\right. \\
w(x, y ; \varepsilon)= & \sum_{p=-\infty}^{\infty} d_{p}(\varepsilon) \exp \left(i\left(\alpha_{p} x-\beta_{w, p}(y-\bar{h})\right)\right) \\
= & \sum_{p=-\infty}^{\infty} \sum_{n=0}^{\infty} d_{p, n} \varepsilon^{n} \exp \left(i\left(\alpha_{p} x-\beta_{w, p}(y-\bar{h})\right)\right) .
\end{aligned}
$$

In light of the non-dimensionalization of the period of the interfaces $(d=2 \pi)$, the parameter $\varepsilon$ is also non-dimensional and measures the "height-to-period" ratio of the profiles.

A careful mathematical analysis of this method in the two-layer case requires analyticity of the interface ${ }^{19,31}$ and we fully anticipate that a similar result can be realized for the $(M+1)$-layer case (this is the subject of current investigation by the authors). However, closely related "transformed" fields can be shown to be analytic in $\varepsilon$ provided that the interface is only Lipschitz (continuous but not necessarily continuously differentiable). It has been our experience that, in practice, profiles as irregular as these can be simulated with excellent results. ${ }^{20,31}$

To determine the $\vec{z}_{p, n}=\left(a_{p, n}, b_{p, n}, c_{p, n}, d_{p, n}\right)^{T}$, we consider the generalization of Eq. (5)

$$
\begin{aligned}
0= & \sum_{p=-\infty}^{\infty} \exp \left(i \alpha_{p} x\right)\left\{a_{p}(\varepsilon) \exp \left(i \beta_{u, p} \varepsilon f\right)\right. \\
& -b_{p}(\varepsilon) \exp \left(-i \beta_{v, p}(\bar{g}+\varepsilon f-\bar{m})\right) \\
& \left.-c_{p}(\varepsilon) \exp \left(i \beta_{v, p}(\bar{g}+\varepsilon f-\bar{m})\right)\right\}
\end{aligned}
$$

$$
\begin{aligned}
0= & \sum_{p=-\infty}^{\infty} \exp \left(i \alpha_{p} x\right)\left\{\left(i \beta_{u, p}-i \alpha_{p} \varepsilon\left(\partial_{x} f\right)\right) a_{p}(\varepsilon)\right. \\
& \times \exp \left(i \beta_{u, p} \varepsilon f\right)-\left(-i \beta_{v, p}-i \alpha_{p} \varepsilon\left(\partial_{x} f\right)\right) b_{p}(\varepsilon) \\
& \times \exp \left(-i \beta_{v, p}(\bar{g}+\varepsilon f-\bar{m})\right)-\left(i \beta_{v, p}-i \alpha_{p} \varepsilon\left(\partial_{x} f\right)\right) \\
& \left.\times c_{p}(\varepsilon) \exp \left(i \beta_{v, p}(\bar{g}+\varepsilon f-\bar{m})\right)\right\}
\end{aligned}
$$

and

$$
\begin{aligned}
\xi(x)= & \sum_{p=-\infty}^{\infty} \exp \left(i \alpha_{p} x\right)\left\{b_{p}(\varepsilon) \exp \left(-i \beta_{v, p}(\bar{h}+\varepsilon s-\bar{m})\right)\right. \\
& +c_{p}(\varepsilon) \exp \left(i \beta_{v, p}(\bar{h}+\varepsilon s-\bar{m})\right) \\
& \left.-\exp \left(-i \beta_{w, p} \varepsilon s\right) d_{p}(\varepsilon)\right\}, \\
\psi(x)= & \sum_{p=-\infty}^{\infty} \exp \left(i \alpha_{p} x\right)\left\{\left(-i \beta_{v, p}-i \alpha_{p} \varepsilon\left(\partial_{x} s\right)\right) b_{p}(\varepsilon)\right. \\
& \times \exp \left(-i \beta_{v, p}(\bar{h}+\varepsilon s-\bar{m})\right)+\left(i \beta_{v, p}-i \alpha_{p} \varepsilon\left(\partial_{x} s\right)\right) \\
& \times c_{p}(\varepsilon) \exp \left(i \beta_{v, p}(\bar{h}+\varepsilon s-\bar{m})\right) \\
& \left.-\left(-i \beta_{w, p}-i \alpha_{p} \varepsilon\left(\partial_{x} s\right)\right) \exp \left(-i \beta_{w, p} \varepsilon s\right) d_{p}(\varepsilon)\right\} .
\end{aligned}
$$

Expanding in Taylor series gives (somewhat complicated) equations for the $\vec{z}_{p, n}$. To give a flavor for this, let us focus upon Eq. (7a)

$$
\begin{aligned}
0= & \sum_{p=-\infty}^{\infty} \exp \left(i \alpha_{p} x\right)\left\{\left(\sum_{n=0}^{\infty} a_{p, n} \varepsilon^{n}\right)\left(\sum_{l=0}^{\infty}\left(i \beta_{u, p}\right)^{l} \frac{(f(x))^{l}}{l !} \varepsilon^{l}\right)\right. \\
& -\left(\sum_{n=0}^{\infty} b_{p, n} \varepsilon^{n}\right)\left(\sum_{l=0}^{\infty}\left(-i \beta_{v, p}\right)^{l} \frac{(f(x))^{l}}{l !} \varepsilon^{l}\right) \\
& \times \exp \left(-i \beta_{v, p}(\bar{g}-\bar{m})\right)-\left(\sum_{n=0}^{\infty} c_{p, n} \varepsilon^{n}\right) \\
& \left.\times\left(\sum_{l=0}^{\infty}\left(i \beta_{v, p}\right)^{l} \frac{(f(x))^{l}}{l !} \varepsilon^{l}\right) \exp \left(i \beta_{v, p}(\bar{g}-\bar{m})\right)\right\} \\
= & \sum_{n=0}^{\infty} \varepsilon^{n} \sum_{p=-\infty}^{\infty} \exp \left(i \alpha_{p} x\right)\left\{\sum_{l=0}^{n} a_{p, n-l}\left(i \beta_{u, p}\right)^{l} \frac{(f(x))^{l}}{l !}\right. \\
& -\sum_{l=0}^{n} b_{p, n-l}\left(-i \beta_{v, p}\right)^{l} \frac{(f(x))^{l}}{l !} \exp \left(-i \beta_{v, p}(\bar{g}-\bar{m})\right) \\
& \left.-\sum_{l=0}^{n} c_{p, n-l}\left(i \beta_{v, p}\right)^{l} \frac{(f(x))^{l}}{l !} \exp \left(i \beta_{v, p}(\bar{g}-\bar{m})\right)\right\}
\end{aligned}
$$

Setting $F_{l}(x)=(f(x))^{l} / l$ ! and denoting its Fourier coefficients by $F_{q, l}$, i.e.,

$$
F_{l}(x)=\sum_{q=-\infty}^{\infty} F_{q, l} e^{i(2 \pi / d) q x}=\sum_{q=-\infty}^{\infty} F_{q, l} e^{i q x}
$$

A. Malcolm and D. Nicholls: Field expansions for multilayered media 
we can further simplify Eq. (8)

$$
\begin{aligned}
0= & \sum_{n=0}^{\infty} \varepsilon^{n} \sum_{l=0}^{n}\left\{\sum_{p=-\infty}^{\infty} \exp \left(i \alpha_{p} x\right) a_{p, n-l}\left(i \beta_{u, p}\right)^{l}\right. \\
& -\sum_{p=-\infty}^{\infty} \exp \left(i \alpha_{p} x\right) b_{p, n-l}\left(-i \beta_{v, p}\right)^{l} \exp \left(-i \beta_{v, p}(\bar{g}-\bar{m})\right) \\
& \left.-\sum_{p=-\infty}^{\infty} \exp \left(i \alpha_{p} x\right) c_{p, n-l}\left(i \beta_{v, p}\right)^{l} \exp \left(i \beta_{v, p}(\bar{g}-\bar{m})\right)\right\} \\
& \times\left(\sum_{q=-\infty}^{\infty} F_{q, l} e^{i q x}\right)
\end{aligned}
$$

so that

$$
\begin{aligned}
0= & \sum_{n=0}^{\infty} \varepsilon^{n} \sum_{l=0}^{n} \sum_{p=-\infty}^{\infty} \exp \left(i \alpha_{p} x\right) \sum_{q=-\infty}^{\infty}\left\{a_{p-q, n-l}\left(i \beta_{u, p-q}\right)^{l}\right. \\
& -b_{p-q, n-l}\left(-i \beta_{v, p-q}\right)^{l} \exp \left(-i \beta_{v, p-q}(\bar{g}-\bar{m})\right) \\
& \left.-c_{p-q, n-l}\left(i \beta_{v, p-q}\right)^{l} \exp \left(i \beta_{v, p-q}(\bar{g}-\bar{m})\right)\right\} F_{q, l} .
\end{aligned}
$$

At order $n=0$ and wavenumber $p$, Eq. (9) amounts to

$$
\begin{aligned}
0= & a_{p, 0}-b_{p, 0} \exp \left(-i \beta_{v, p}(\bar{g}-\bar{m})\right) \\
& -c_{p, 0} \exp \left(i \beta_{v, p}(\bar{g}-\bar{m})\right)
\end{aligned}
$$

since $F_{q, 0}=1$ only if $q=0$; this is simply the first equation in Eq. (6). For orders $n>0$, we find that Eq. (9) implies

$$
\begin{aligned}
& a_{p, n}-b_{p, n} \exp \left(-i \beta_{v, p}(\bar{g}-\bar{m})\right) \\
& \quad-c_{p, n} \exp \left(i \beta_{v, p}(\bar{g}-\bar{m})\right)=\rho_{p, n},
\end{aligned}
$$

where $\rho_{p, n}$ are the Fourier coefficients of the function

$$
\begin{aligned}
\rho_{n}(x)= & \sum_{l=1}^{n} \sum_{p=-\infty}^{\infty} \exp \left(i \alpha_{p} x\right) \sum_{q=-\infty}^{\infty}\left\{-a_{p-q, n-l}\left(i \beta_{u, p-q}\right)^{l}\right. \\
& +b_{p-q, n-l}\left(-i \beta_{v, p-q}\right)^{l} \exp \left(-i \beta_{v, p-q}(\bar{g}-\bar{m})\right) \\
& \left.+c_{p-q, n-l}\left(i \beta_{v, p-q}\right)^{l} \exp \left(i \beta_{v, p-q}(\bar{g}-\bar{m})\right)\right\} F_{q, l} .
\end{aligned}
$$

This can be repeated for the other equations in Eq. (7). At order $n=0$, this delivers exactly Eq. (6), the equations in the flat interface configuration. For order $n>0$, the developments are a little more involved but they result in $\mathcal{A}_{p} \vec{z}_{p, n}=\vec{R}_{p, n}$, where $\vec{R}_{p, n}$ is the Fourier coefficients of the right hand side $\vec{R}_{n}$. In more detail, $\vec{R}_{n}^{(1)}=\rho_{p, n}$,

$$
\begin{aligned}
\vec{R}_{n}^{(2)}= & \sum_{p=-\infty}^{\infty} \exp \left(i \alpha_{p} x\right) \sum_{l=1}^{n} \sum_{q=-\infty}^{\infty}-\left[F_{q, l}\left(i \beta_{u, p-q}\right)^{2}\right. \\
& \left.-\left(\partial_{x} f\right) F_{q, l-1}\left(i \alpha_{p-q}\right)\right]\left(i \beta_{u, p-q}\right)^{l-1} a_{p-q, n-l} \\
& +\left[F_{q, l}\left(-i \beta_{v, p-q}\right)^{2}-\left(\partial_{x} f\right) F_{q, l-1}\left(i \alpha_{p-q}\right)\right]\left(-i \beta_{v, p-q}\right)^{l-1} \\
& \times \exp \left(-i \beta_{v, p-q}(\bar{g}-\bar{m})\right) b_{p-q, n-l} \\
& +\left[F_{q, l}\left(i \beta_{v, p-q}\right)^{2}-\left(\partial_{x} f\right) F_{q, l-1}\left(i \alpha_{p-q}\right)\right]\left(i \beta_{v, p-q}\right)^{l-1} \\
& \times \exp \left(i \beta_{v, p-q}(\bar{g}-\bar{m})\right) c_{p-q, n-l},
\end{aligned}
$$

and

$$
\begin{aligned}
\vec{R}_{n}^{(3)}= & \sum_{p=-\infty}^{\infty} \exp \left(i \alpha_{p} x\right) \sum_{l=1}^{n} \sum_{q=-\infty}^{\infty}-b_{p-q, n-l}\left(-i \beta_{v, p-q}\right)^{l} \\
& \times S_{q, l} \exp \left(-i \beta_{v, p-q}(\bar{g}-\bar{m})\right)-c_{p-q, n-l}\left(i \beta_{v, p-q}\right)^{l} S_{q, l} \\
& \times \exp \left(i \beta_{v, p-q}(\bar{g}-\bar{m})\right)+d_{p-q, n-l}\left(-i \beta_{w, p-q}\right)^{l} S_{q, l}
\end{aligned}
$$

and

$$
\begin{aligned}
\vec{R}_{n}^{(4)}= & \sum_{p=-\infty}^{\infty} \exp \left(i \alpha_{p} x\right) \sum_{l=1}^{n} \sum_{q=-\infty}^{\infty} \\
& -\left[S_{q, l}\left(-i \beta_{v, p-q}\right)^{2}-\left(\partial_{x} s\right) S_{q, l-1}\left(i \alpha_{p-q}\right)\right]\left(-i \beta_{v, p-q}\right)^{l-1} \\
& \times \exp \left(-i \beta_{v, p-q}(\bar{g}-\bar{m})\right) b_{p-q, n-l}-\left[S_{q, l}\left(i \beta_{v, p-q}\right)^{2}\right. \\
& \left.-\left(\partial_{x} s\right) S_{q, l-1}\left(i \alpha_{p-q}\right)\right]\left(i \beta_{v, p-q}\right)^{l-1} \\
& \times \exp \left(i \beta_{v, p-q}(\bar{g}-\bar{m})\right) c_{p-q, n-l}+\left[S_{q, l}\left(-i \beta_{w, p-q}\right)^{2}\right. \\
& \left.-\left(\partial_{x} s\right) S_{q, l-1}\left(i \alpha_{p-q}\right)\right]\left(-i \beta_{w, p-q}\right)^{l-1} S_{q, l}, d_{p-q, n-l}
\end{aligned}
$$

where a negative Taylor index $n$ in any of $\left\{a_{p, n}, b_{p, n}, c_{p, n}\right.$, $\left.d_{p, n}\right\}$ is understood to be zero. In these equations, we define $S_{l, q}$ as the Fourier coefficients of $S_{l}(x)=(s(x))^{l} / l$, , i.e.,

$$
S_{l}(x)=\sum_{q=-\infty}^{\infty} S_{q, l} e^{i(2 \pi / d) q x}=\sum_{q=-\infty}^{\infty} S_{q, l} e^{i q x} .
$$

\section{FIELD EXPANSIONS: $(M+1)$ LAYERS}

In the general $(M+1)$-layer case $(M>1)$, we consider interfaces specified at $y=a^{(m)}+g^{(m)}(x)$ for $1 \leq m \leq M$. Defining the domains

$$
\begin{aligned}
S^{(0)}= & \left\{(x, y) \mid y>a^{(1)}+g^{(1)}(x)\right\}, \\
S^{(m)}= & \left\{(x, y) \mid a^{(m+1)}+g^{(m+1)}(x)<y<a^{(m)}+g^{(m)}(x)\right\}, \\
& 1 \leq m \leq M-1, \\
S^{(M)}= & \left\{(x, y) \mid y<a^{(M)}+g^{(M)}(x)\right\},
\end{aligned}
$$

with normals $N^{(m)}=\left(-\partial_{x} g^{(m)}, 1\right)^{T}$, the scattered field $v$ satisfies the system of Helmholtz equations [cf. Eqs. (2a), (2c), and (2e)]

$$
\Delta v^{(m)}+\left(k^{(m)}\right)^{2} v^{(m)}=0 \quad \text { in } S^{(m)}, \quad 0 \leq m \leq M,
$$

where $v^{(m)}$ is $v$ restricted to $S^{(m)}$. For incident radiation of the form (1), one has $k^{(m)}=\omega / c_{m}$. These must be supplemented with the general boundary conditions

$$
\begin{aligned}
& {\left[v^{(m-1)}-v^{(m)}\right]_{y=\alpha^{(m)}+g^{(m)}}=\xi^{(m)}, \quad 1 \leq m \leq M,} \\
& {\left[\partial_{N^{(m)}} v^{(m-1)}-\partial_{N^{(m)}} v^{(m)}\right]_{y=\alpha^{(m)}+g^{(m)}}=\psi^{(m)},} \\
& 1 \leq m \leq M,
\end{aligned}
$$

cf. (2d) and (2g), where $\xi^{(m)} \equiv 0, \psi^{(m)} \equiv 0$ for $m \neq M$, for a plane-wave incident from below; we briefly discuss other 
incident fields in Sec. IV B. Again, the solutions of these Helmholtz problems outside the grooves are

$$
\begin{aligned}
v^{(m)}(x, y)= & \sum_{p=-\infty}^{\infty} d_{p}^{(m)} \exp \left(i\left(\alpha_{p} x-\beta_{p}^{(m)}\left(y-\bar{a}^{(m)}\right)\right)\right) \\
& +\sum_{p=-\infty}^{\infty} u_{p}^{(m)} \exp \left(i\left(\alpha_{p} x+\beta_{p}^{(m)}\left(y-\bar{a}^{(m)}\right)\right)\right),
\end{aligned}
$$

where the $\bar{a}^{(m)}$ are the mid-levels of each layer

$$
\bar{a}^{(0)}=a^{(1)}, \quad \bar{a}^{(m)}=\frac{1}{2}\left(a^{(m)}+a^{(m+1)}\right), \quad \bar{a}^{(M)}=a^{(M)},
$$

and

$$
\beta_{p}^{(m)}= \begin{cases}\sqrt{\left(k^{(m)}\right)^{2}-\alpha_{p}^{2}} & \alpha_{p}^{2}<\left(k^{(m)}\right)^{2} \\ i \sqrt{\alpha_{p}^{2}-\left(k^{(m)}\right)^{2}} & \alpha_{p}^{2}>\left(k^{(m)}\right)^{2}\end{cases}
$$

The OWC can be enforced by choosing $d_{p}^{(0)}=u_{p}^{(M)} \equiv 0$. To determine the other coefficients, we appeal to the boundary conditions at the interfaces $y=a^{(m)}+g^{(m)}(x)$, Eq. (10).

\section{A. Trivial interfaces}

For the case of flat (trivial) interfaces, i.e., $g^{(m)} \equiv 0$ for $1 \leq m \leq M$, the Dirichlet condition (10a) coupled to the representation (11) states that

$$
\begin{aligned}
\xi^{(m)}(x)=\sum_{p=-\infty}^{\infty} & \left\{d_{p}^{(m-1)} \exp \left(-i \beta_{p}^{(m-1)}\left(a^{(m)}-\bar{a}^{(m-1)}\right)\right)\right. \\
& +u_{p}^{(m-1)} \exp \left(i \beta_{p}^{(m-1)}\left(a^{(m)}-\bar{a}^{(m-1)}\right)\right) \\
& -d_{p}^{(m)} \exp \left(-i \beta_{p}^{(m)}\left(a^{(m)}-\bar{a}^{(m)}\right)\right) \\
& \left.-u_{p}^{(m)} \exp \left(i \beta_{p}^{(m)}\left(a^{(m)}-\bar{a}^{(m)}\right)\right)\right\} \exp \left(i \alpha_{p} x\right) .
\end{aligned}
$$

At this point, we switch to a more concise, and we feel more elegant, notation for the boundary conditions in terms of
Fourier multipliers. In this new notation, the Dirichlet condition (12) is

$$
\begin{aligned}
\xi^{(m)}= & D^{(m, m-1)} d^{(m-1)}+U^{(m, m-1)} u^{(m-1)} \\
& -D^{(m, m)} d^{(m)}-U^{(m, m)} u^{(m)}
\end{aligned}
$$

(recall that $d^{(0)}=u^{(M)} \equiv 0$ ), and, by similar calculations, the Neumann condition (10b) becomes

$$
\begin{aligned}
\psi^{(m)}= & -B^{(m-1)} D^{(m, m-1)} d^{(m-1)}+B^{(m-1)} U^{(m, m-1)} u^{(m-1)} \\
& +B^{(m)} D^{(m, m)} d^{(m)}-B^{(m)} U^{(m, m)} u^{(m)}
\end{aligned}
$$

In these formulas, we use the Fourier multipliers

$$
\begin{aligned}
D^{(m, l)}[\zeta] & =\sum_{p=-\infty}^{\infty} \exp \left(-i \beta_{p}^{(l)}\left(a^{(m)}-\bar{a}^{(l)}\right)\right) \hat{\zeta}_{p} \exp \left(i \alpha_{p} x\right) \\
U^{(m, l)}[\zeta] & =\sum_{p=-\infty}^{\infty} \exp \left(i \beta_{p}^{(l)}\left(a^{(m)}-\bar{a}^{(l)}\right)\right) \hat{\zeta}_{p} \exp \left(i \alpha_{p} x\right) \\
B^{(m)}[\zeta] & =\sum_{p=-\infty}^{\infty}\left(i \beta_{p}^{(m)}\right) \hat{\zeta}_{p} \exp \left(i \alpha_{p} x\right)
\end{aligned}
$$

where the first two are "order zero" and the latter is "order one." We recall that a Fourier multiplier of order $j$ maps a function with $(s+j)$-many $L^{2}$ derivatives to a function with $s$-many $L^{2}$ derivatives. ${ }^{35}$ Thus, the operators $D^{(m, l)}, U^{(m, l)}$ "take no derivatives," while $B^{(m)}$, like the classical derivative, "takes one derivative."

Thus, we have the following system of linear equations to solve

$$
\mathcal{A} \vec{z}=\vec{r}
$$

where

$$
\begin{aligned}
& \vec{z}=\left(u^{(0)}, d^{(1)}, u^{(1)}, \ldots, d^{(M-1)}, u^{(M-1)}, d^{(M)}\right)^{T}, \\
& \vec{r}=\left(\xi^{(1)}, \psi^{(1)}, \xi^{(2)}, \psi^{(2)}, \ldots, \xi^{(M)}, \psi^{(M)}\right)^{T},
\end{aligned}
$$

and

$$
\mathcal{A}=\left(\begin{array}{cccccc}
U_{1,0} & -D_{1,1} & -U_{1,1} & 0 & 0 & 0 \\
B_{0} U_{1,0} & B_{1} D_{1,1} & -B_{1} U_{1,1} & 0 & 0 & 0 \\
0 & D_{2,1} & U_{2,1} & -D_{2,2} & -U_{2,2} & 0 \\
0 & -B_{1} D_{2,1} & B_{1} U_{2,1} & B_{2} D_{2,2} & -B_{2} U_{2,2} & 0 \\
\vdots & & & & & \vdots \\
0 & 0 & 0 & D_{M, M-1} & U_{M, M-1} & -D_{M, M} \\
0 & 0 & 0 & -B_{M-1} D_{M, M-1} & B_{M-1} U_{M, M-1} & B_{M} D_{M, M}
\end{array}\right)
$$

Of course all of these operators are diagonalized by the Fourier transform so we can solve, wavenumber-by-wavenumber, the systems

$$
\mathcal{A}_{p} \vec{z}_{p}=\vec{r}_{p}
$$

where 


$$
\begin{aligned}
& \vec{z}_{p}=\left(u_{p}^{(0)}, d_{p}^{(1)}, u_{p}^{(1)}, \ldots, d_{p}^{(M-1)}, u_{p}^{(M-1)}, d_{p}^{(M)}\right)^{T}, \\
& \vec{r}_{p}=\left(\hat{\xi}_{p}^{(1)}, \hat{\psi}_{p}^{(1)}, \hat{\xi}_{p}^{(2)}, \hat{\psi}_{p}^{(2)}, \ldots, \hat{\xi}_{p}^{(M)}, \hat{\psi}_{p}^{(M)}\right)^{T},
\end{aligned}
$$

and $\mathcal{A}_{p}$ is penta-diagonal

$$
\begin{aligned}
\left(\mathcal{A}_{p}\right)_{2 m-1,2 m-2} & =\left(D_{m, m-1}\right)_{p}=\exp \left(-i \beta_{p}^{(m-1)}\left(a^{(m)}-\bar{a}^{(m-1)}\right)\right), \\
\left(\mathcal{A}_{p}\right)_{2 m-1,2 m-1} & =\left(U_{m, m-1}\right)_{p}=\exp \left(i \beta_{p}^{(m-1)}\left(a^{(m)}-\bar{a}^{(m-1)}\right)\right), \\
\left(\mathcal{A}_{p}\right)_{2 m-1,2 m} & =-\left(D_{m, m}\right)_{p}=-\exp \left(-i \beta_{p}^{(m)}\left(a^{(m)}-\bar{a}^{(m)}\right)\right), \\
\left(\mathcal{A}_{p}\right)_{2 m-1,2 m+1} & =-\left(U_{m, m}\right)_{p}=-\exp \left(i \beta_{p}^{(m)}\left(a^{(m)}-\bar{a}^{(m)}\right)\right), \\
\left(\mathcal{A}_{p}\right)_{2 m, 2 m-2} & =-\left(B_{m-1} D_{m, m-1}\right)_{p} \\
& =-\left(i \beta_{p}^{(m-1)}\right) \exp \left(-i \beta_{p}^{(m-1)}\left(a^{(m)}-\bar{a}^{(m-1)}\right)\right), \\
\left(\mathcal{A}_{p}\right)_{2 m, 2 m-1} & =\left(B_{m-1} U_{m, m-1}\right)_{p} \\
& =\left(i \beta_{p}^{(m-1)}\right) \exp \left(i \beta_{p}^{(m-1)}\left(a^{(m)}-\bar{a}^{(m-1)}\right)\right), \\
\left(\mathcal{A}_{p}\right)_{2 m, 2 m}= & \left(B_{m} D_{m, m}\right)_{p}=\left(i \beta_{p}^{(m)}\right) \exp \left(-i \beta_{p}^{(m)}\left(a^{(m)}-\bar{a}^{(m)}\right)\right), \\
\left(\mathcal{A}_{p}\right)_{2 m, 2 m+1} & =-\left(B_{m} U_{m, m}\right)_{p} \\
& =-\left(i \beta_{p}^{(m)}\right) \exp \left(i \beta_{p}^{(m)}\left(a^{(m)}-\bar{a}^{(m)}\right)\right),
\end{aligned}
$$

for $1 \leq m \leq M$; formulas which produce indices outside the range $1 \leq m \leq M$ [i.e., $\left(\mathcal{A}_{p}\right)_{1,0}$ and $\left(\mathcal{A}_{p}\right)_{M, M+1}$ ] are ignored. Since the system (15) is penta-diagonal it can be solved quickly [in time $\mathcal{O}(M)$ ] using the standard techniques. This is the crucial observation which enables our accelerated method for couplers with non-trivial interface shapes.

\section{B. Non-trivial interfaces}

To address the case of non-trivial interfaces, we can again use the representation Eq. (11) together with $d_{p}^{(0)}=u_{p}^{(M)} \equiv 0$. The Dirichlet and Neumann conditions remain as (13) and (14), respectively; however, we must now understand the operators $D^{(m, l)}$ and $U^{(m, l)}$ as $g^{(m)}$ dependent

$$
\begin{aligned}
D^{(m, l)}\left(g^{(m)}\right)[\zeta]= & \sum_{p=-\infty}^{\infty} \exp \left(-i \beta_{p}^{(l)}\left(a^{(m)}+g^{(m)}-\bar{a}^{(l)}\right)\right) \\
& \times \hat{\zeta}_{p} \exp \left(i \alpha_{p} x\right), \\
U^{(m, l)}\left(g^{(m)}\right)[\zeta]= & \sum_{p=-\infty}^{\infty} \exp \left(i \beta_{p}^{(l)}\left(a^{(m)}+g^{(m)}-\bar{a}^{(l)}\right)\right) \\
& \times \hat{\zeta}_{p} \exp \left(i \alpha_{p} x\right) .
\end{aligned}
$$

Following our previous developments, we pursue the FE method ${ }^{20}$ beginning with the assumption that the interfaces $g^{(m)}$ are deviations of the trivial interface case, and that these deviations can be parametrized by the single variable $\varepsilon$, i.e., $g^{(m)}(x)=\varepsilon f^{(m)}(x)$. A generalization of the work of Nicholls and Reitich ${ }^{31}$ will show that the fields $v^{(m)}$ depend analytically upon $\varepsilon$ so that the expansions,

$$
\begin{aligned}
v^{(m)}= & v^{(m)}(x, y ; \varepsilon)=\sum_{p=-\infty}^{\infty} d_{p}^{(m)}(\varepsilon) \exp \left(i\left(\alpha_{p} x-\beta_{p}^{(m)}\left(y-\bar{a}^{(m)}\right)\right)\right) \\
& +u_{p}^{(m)}(\varepsilon) \exp \left(i\left(\alpha_{p} x+\beta_{p}^{(m)}\left(y-\bar{a}^{(m)}\right)\right)\right) \\
= & \sum_{p=-\infty}^{\infty} \sum_{n=0}^{\infty}\left\{d_{p, n}^{(m)} \exp \left(i\left(\alpha_{p} x-\beta_{p}^{(m)}\left(y-\bar{a}^{(m)}\right)\right)\right)\right. \\
& \left.+u_{p, n}^{(m)} \exp \left(i\left(\alpha_{p} x+\beta_{p}^{(m)}\left(y-\bar{a}^{(m)}\right)\right)\right)\right\} \varepsilon^{n},
\end{aligned}
$$

can be rigorously justified provided that the $f^{(m)}$ are sufficiently small and smooth. To find the coefficients $d_{p, n}^{(m)}$ and $u_{p, n}^{(m)}$ we use the conditions (13) and (14) with the dependence of $\varepsilon$ emphasized:

$$
\begin{aligned}
\xi^{(m)}= & D^{(m, m-1)}(\varepsilon) d^{(m-1)}(\varepsilon)+U^{(m, m-1)}(\varepsilon) u^{(m-1)}(\varepsilon) \\
& -D^{(m, m)}(\varepsilon) d^{(m)}(\varepsilon)-U^{(m, m)}(\varepsilon) u^{(m)}(\varepsilon),
\end{aligned}
$$

and

$$
\begin{aligned}
\psi^{(m)}= & D^{(m, m-1)}(\varepsilon)\left(-B^{(m-1)}-\varepsilon\left(\partial_{x} f\right) \partial_{x}\right) d^{(m-1)}(\varepsilon) \\
& +U^{(m, m-1)}(\varepsilon)\left(B^{(m-1)}-\varepsilon\left(\partial_{x} f\right) \partial_{x}\right) u^{(m-1)}(\varepsilon) \\
& -D^{(m, m)}(\varepsilon)\left(-B^{(m)}-\varepsilon\left(\partial_{x} f\right) \partial_{x}\right) d^{(m)}(\varepsilon) \\
& -U^{(m, m)}(\varepsilon)\left(B^{(m)}-\varepsilon\left(\partial_{x} f\right) \partial_{x}\right) u^{(m)}(\varepsilon) .
\end{aligned}
$$

To use these, we need the Taylor expansions

$$
\begin{aligned}
& D^{(m, l)}\left(\varepsilon f^{(m)}\right)=\sum_{n=0}^{\infty} D_{n}^{(m, l)}\left(f^{(m)}\right) \varepsilon^{n}, \\
& U^{(m, l)}\left(\varepsilon f^{(m)}\right)=\sum_{n=0}^{\infty} U_{n}^{(m, l)}\left(f^{(m)}\right) \varepsilon^{n},
\end{aligned}
$$

where $D_{0}^{(m, l)}=D^{(m, l)}(0), U_{0}^{(m, l)}=U^{(m, l)}(0)$,

$$
\begin{gathered}
D_{n}^{(m, l)}\left(f^{(m)}\right)[\zeta]=F_{n}^{(m)}\left(-B^{(l)}\right)^{n} D_{0}^{(m, l)} \zeta, \\
U_{n}^{(m, l)}\left(f^{(m)}\right)[\zeta]=F_{n}^{(m)}\left(B^{(l)}\right)^{n} U_{0}^{(m, l)} \zeta,
\end{gathered}
$$

and $F_{n}^{(m)}=\left(\left(f^{(m)}\right)^{n}\right) / n$ !. With these, we can realize the following recursions from Eqs. (18) and (19): At order zero, we have

$$
\begin{aligned}
& D_{0}^{(m, m-1)} d_{0}^{(m-1)}+U_{0}^{(m, m-1)} u_{0}^{(m-1)}-D_{0}^{(m, m)} d_{0}^{(m)} \\
& \quad-U_{0}^{(m, m)} u_{0}^{(m)}=\xi^{(m)}, \\
& -B^{(m-1)} D_{0}^{(m, m-1)} d_{0}^{(m-1)}+B^{(m-1)} U_{0}^{(m, m-1)} u_{0}^{(m-1)} \\
& \quad+B^{(m)} D_{0}^{(m, m)} d_{0}^{(m)}-B^{(m)} U_{0}^{(m, m)} u_{0}^{(m)}=\psi^{(m)},
\end{aligned}
$$

which, of course, is simply Eqs. (13) and (14) and we can solve this system, for each wavenumber, in linear time in $M$. For $n>0$, we obtain

$$
\begin{aligned}
& D_{0}^{(m, m-1)} d_{n}^{(m-1)}+U_{0}^{(m, m-1)} u_{n}^{(m-1)}-D_{0}^{(m, m)} d_{n}^{(m)} \\
& \quad-U_{0}^{(m, m)} u_{n}^{(m)}=Q_{n}^{(m)}, \\
& -B^{(m-1)} D_{0}^{(m, m-1)} d_{n}^{(m-1)}+B^{(m-1)} U_{0}^{(m, m-1)} u_{n}^{(m-1)} \\
& \quad+B^{(m)} D_{0}^{(m, m)} d_{n}^{(m)}-B^{(m)} U_{0}^{(m, m)} u_{n}^{(m)}=T_{n}^{(m)},
\end{aligned}
$$

where

$$
\begin{aligned}
Q_{n}^{(m)}= & -\left\{\sum_{l=1}^{n} D_{l}^{(m, m-1)} d_{n-l}^{(m-1)}+U_{l}^{(m, m-1)} u_{n-l}^{(m-1)}\right. \\
& \left.-D_{l}^{(m, m)} d_{n-l}^{(m)}-U_{l}^{(m, m)} u_{n-l}^{(m)}\right\},
\end{aligned}
$$




$$
\begin{aligned}
T_{n}^{(m)}= & -\left\{\sum_{l=1}^{n}-B^{(m-1)} D_{l}^{(m, m-1)} d_{n-l}^{(m-1)}\right. \\
& -\left(\partial_{x} f\right) D_{l-1}^{(m, m-1)} \partial_{x} d_{n-l}^{(m-1)}+B^{(m-1)} U_{l}^{(m, m-1)} u_{n-l}^{(m-1)} \\
& -\left(\partial_{x} f\right) U_{l-1}^{(m, m-1)} \partial_{x} u_{n-l}^{(m-1)}+B^{(m)} D_{l}^{(m, m)} d_{n-l}^{(m)} \\
& +\left(\partial_{x} f\right) D_{l-1}^{(m, m)} \partial_{x} d_{n-l}^{(m)}-B^{(m)} U_{l}^{(m, m)} u_{n-l}^{(m)} \\
& \left.+\left(\partial_{x} f\right) U_{l-1}^{(m, m)} \partial_{x} u_{n-l}^{(m)}\right\}
\end{aligned}
$$

are known from the solution at previous orders. Using the calculation above in Eq. (20), we can simplify the terms in Eq. (23)

$$
\begin{aligned}
Q_{n}^{(m)}= & -\left\{\sum_{l=1}^{n} F_{l}^{(m)}\left(-B^{(m-1)}\right)^{l} D_{0}^{(m, m-1)} d_{n-l}^{(m-1)}+F_{l}^{(m)}\right. \\
& \times\left(B^{(m-1)}\right)^{l} U_{0}^{(m, m-1)} u_{n-l}^{(m-1)}-F_{l}^{(m)}\left(-B^{(m)}\right)^{l} \\
& \left.D_{0}^{(m, m)} d_{n-l}^{(m)}-F_{l}^{(m)}\left(B^{(m)}\right)^{l} U_{0}^{(m, m)} u_{n-l}^{(m)}\right\} \\
T_{n}^{(m)}= & -\left\{\sum_{l=1}^{n} F_{l}^{(m)}\left(-B^{(m-1)}\right)^{l+1} D_{0}^{(m, m-1)} d_{n-l}^{(m-1)}\right. \\
& -\left(\partial_{x} f\right) F_{l-1}^{(m)} \partial_{x}\left(-B^{(m-1)}\right)^{l-1} D_{0}^{(m, m-1)} d_{n-l}^{(m-1)} \\
& +F_{l}^{(m)}\left(B^{(m-1)}\right)^{l+1} U_{0}^{(m, m-1)} u_{n-l}^{(m-1)} \\
& -\left(\partial_{x} f\right) F_{l-1}^{(m)} \partial_{x}\left(B^{(m-1)}\right)^{l-1} U_{0}^{(m, m-1)} u_{n-l}^{(m-1)} \\
& -F_{l}^{(m)}\left(-B^{(m)}\right)^{l+1} D_{0}^{(m, m)} d_{n-l}^{(m)} \\
& +\left(\partial_{x} f\right) F_{l-1}^{(m)} \partial_{x}\left(-B^{(m)}\right)^{l-1} D_{0}^{(m, m)} d_{n-l}^{(m)} \\
& -F_{l}^{(m)}\left(B^{(m)}\right)^{l+1} U_{0}^{(m, m)} u_{n-l}^{(m)} \\
& \left.+\left(\partial_{x} f\right) F_{l-1}^{(m)} \partial_{x}\left(B^{(m)}\right)^{l-1} U_{0}^{(m, m)} u_{n-l}^{(m)}\right\}
\end{aligned}
$$

Our key observation is that Eq. (22) is simply Eq. (15) with the right hand side replaced by

$$
\vec{R}_{n}=\left(Q_{n}^{(1)}, T_{n}^{(1)}, Q_{n}^{(2)}, T_{n}^{(2)}, \ldots, Q_{n}^{(M)}, T_{n}^{(M)}\right)^{T}
$$

and can, therefore, be solved rapidly via standard techniques. In fact, a quick count of operations yields a work estimate of $\mathcal{O}\left(M N^{2} N_{x} \log \left(N_{x}\right)\right)$ if we truncate our Fourier-Taylor series $\left\{d_{p, n}^{(m)}, u_{p, n}^{(m)}\right\}$ after $N_{x}$ modes and $N$ orders. More precisely, at every Taylor order $0 \leq n \leq N$, and every wavenumber $-N_{x} /$ $2 \leq p \leq N_{x} / 2-1$, we solve a linear system of size $M$ in linear time. To form the right hand sides of the linear system requires fast convolutions [via the FFT algorithm in time $\left.\mathcal{O}\left(N_{x} \log \left(N_{x}\right)\right)\right]$ and a sum of length $n$ (over indices $0 \leq l \leq n-1)$.

This is to be contrasted with the work of Wilcox et al. ${ }^{3}$ who solve these layer problems sequentially using the twolayer solver of Bruno and Reitich. ${ }^{20}$ For instance, in the three-layer case outlined in Sec. II, incident radiation from below results in a field scattered by the lowest layer at $y=\bar{h}+h(x)$ which is partially reflected downward and partially transmitted upward. Wilcox et al compute these at the interface $y=\bar{h}+h(x)$ with a two-layer solver, but now must account for the fact that the transmitted field will interact with the layer at $y=\bar{g}+g(x)$ producing a scattered field transmitting further up the structure and a reflected field which travels back to $y=\bar{h}+h(x)$. This transmitted/ reflected pair is computed in the second "bounce," but this procedure continues ad infinitum (albeit with decreasing amplitude in the inner part of the structure at every bounce). So, to compare with the cost of our new approach, that of Wilcox et al. is $\mathcal{O}\left(B N^{2} N_{x} \log \left(N_{x}\right)\right)$, where $B$ is the number of bounces required to reach a certain error tolerance. These authors report values of $B$ in the range of 500-1000 for configurations with $M=2$ interfaces, clearly disadvantaged with respect to our new approach.

\section{NUMERICAL VALIDATION}

In this section, we show how the algorithms we have described can be used in multi-layer simulations. In brief, the method discussed above can be summarized as a Fourier Collocation $^{36} /$ Taylor method ${ }^{29}$ enhanced by Padé summation techniques. ${ }^{37}$ In more detail, we approximate the fields $v^{(m)}$, cf. Eq. (17), by

$$
\begin{aligned}
v^{\left(m, N_{x}, N\right)}= & \sum_{p=-N_{x} / 2}^{N_{x} / 2-1} \sum_{n=0}^{N}\left\{d_{p, n}^{(m)} \exp \left(i\left(\alpha_{p} x-\beta_{p}^{(m)}\left(y-\bar{a}^{(m)}\right)\right)\right)\right. \\
& \left.+u_{p, n}^{(m)} \exp \left(i\left(\alpha_{p} x+\beta_{p}^{(m)}\left(y-\bar{a}^{(m)}\right)\right)\right)\right\} \varepsilon^{n},
\end{aligned}
$$

which are then inserted into Eq. (22). At this point, the only considerations are how the convolution products present in the right hand sides, $\left\{Q_{n}, R_{n}\right\}$ cf. Eq. (23), are to be computed, and how the sum in $\varepsilon$ is to be formed. For the former, we utilize the discrete Fourier transform accelerated by the fast Fourier transform algorithm, ${ }^{36}$ and for the latter, we approximate the truncated order $N$ Taylor series by its $N / 2-N / 2$ Padé approximant. To make all of this absolutely clear, we recall $^{37}$ that if an analytic function,

$$
F(\varepsilon)=\sum_{n=0}^{\infty} F_{n} \varepsilon^{n},
$$

is approximated by its order $N$ truncation,

$$
F^{N}(\varepsilon)=\sum_{n=0}^{N} F_{n} \varepsilon^{n},
$$

then the convergence of $F^{N}$ to $F$ can typically be greatly enhanced with the use of the $P-Q$ Padé approximant

$$
[P / Q](\varepsilon)=\frac{\sum_{l=0}^{P} a_{l} \varepsilon^{l}}{1+\sum_{m=1}^{Q} b_{m} \varepsilon^{m}} \approx F^{N}(\varepsilon),
$$

where $P+Q=N$. Algorithms for the computation of the $\left\{a_{l}\right\}$ and $\left\{b_{m}\right\}$ are readily available ${ }^{37}$ and easy to implement. 
TABLE I. Relative error (maximum norm) versus number of Taylor series terms retained, cf. Eq. (25), in a simulation of scattering by a three-layer structure. Physical parameters are reported in Eq. (27) while the numerical parameters were $N_{x}=128$ and $N_{\max }=24$.

\begin{tabular}{ll}
\hline \hline$N$ & \multicolumn{1}{c}{ Relative error } \\
\hline 0 & 0.223127 \\
2 & 0.00698624 \\
4 & 0.000291307 \\
6 & $1.27346 \times 10^{-5}$ \\
8 & $5.49206 \times 10^{-7}$ \\
10 & $2.76567 \times 10^{-8}$ \\
12 & $2.25986 \times 10^{-9}$ \\
14 & $2.04645 \times 10^{-10}$ \\
16 & $6.27159 \times 10^{-11}$ \\
\hline \hline
\end{tabular}

\section{A. Convergence}

To verify our code, we compare with a configuration in which exact solutions are readily available; the specific solution we choose is unphysical; however, it does provide definitive evidence for the convergence of our scheme. We note that in each of the layers there are solutions of the form

$$
\begin{aligned}
v^{(m)}(x, y)= & A_{\mathrm{up}}^{(m)} e^{i\left(\alpha_{p} x+\beta_{p}^{(m)} y\right)}+A_{\mathrm{down}}^{(m)} e^{i\left(\alpha_{p} x-\beta_{p}^{(m)} y\right)}, \\
& 0 \leq m \leq M,
\end{aligned}
$$

for any integer $p$. Enforcing $A_{\mathrm{down}}^{(0)}=A_{\mathrm{up}}^{(M)}=0$ and choosing the rest of the $A_{\text {up }}^{(m)}$ and $A_{\text {down }}^{(m)}$ provides us with an easily computed and manipulated exact solution (which is neither generated by plane-waves nor continuous across layer interfaces). With these choices and Eq. (26), the jumps in Dirichlet data, $\xi^{(m)}$, and Neumann data, $\psi^{(m)}$, cf. Eq. (10), can be readily computed.

To verify our implementation we consider the threelayer case

$$
\begin{aligned}
& \beta_{u}=1.1, \quad \beta_{v}=2.2, \quad \beta_{w}=3.3, \\
& \bar{g}=-1, \quad g(x)=\varepsilon \cos (x), \quad \bar{h}=1, \quad h(x)=\varepsilon \sin (2 x),
\end{aligned}
$$

$\varepsilon=0.1$, and

$$
p=0, \quad A_{\text {up }}^{(0)}=A_{\text {up }}^{(1)}=1, \quad A_{\text {down }}^{(1)}=A_{\text {down }}^{(2)}=1,
$$

in Eq. (26). For numerical parameters, we selected $N_{x}=128$ and $N=24$. In Table I, we report on the relative error in the maximum (supremum or $L^{\infty}$ ) norm in the entire computational domain $[0,2 \pi] \times\left[y_{\min }, y_{\max }\right]$ (we selected $y_{\min }=-2$ and $y_{\max }=2$ ).

From this data, we see that our new algorithm can produce spectrally accurate solutions throughout all layers and at every wavenumber $p$.

\section{B. Plane-wave and point-source scattering}

We now present the results of the two numerical experiments featuring three- and five-layer structures. In both of these experiments, we have chosen $d=2 \pi$ periodic interfaces with $\alpha=0.1$. In the three-layer case, we have selected

$$
\begin{aligned}
& \beta_{u}=1.1, \quad \beta_{v}=2.2, \quad \beta_{w}=3.3, \\
& \bar{g}=-1, \quad g(x)=\varepsilon \cos (x), \quad \bar{h}=1, \quad h(x)=\varepsilon \sin (2 x),
\end{aligned}
$$

cf. Eq. (28), and $\varepsilon=0.1$. For numerical parameters, we selected $N_{x}=128$ and $N=24$. To verify the accuracy of our simulations, we consider the "energy defect" in our solution. For a lossless structure like the ones considered in this paper, it is known that the total energy is conserved..$^{33}$ This principle can be stated precisely in terms of the efficiencies, $e_{p}^{(j)},{ }^{33}$

$$
\begin{gathered}
e_{p}^{(0)}=\frac{\left|u_{p}^{(0)^{2}}\right| \beta_{p}^{(0)}}{\beta} \quad p \in U^{(0)}=\left\{p \mid \alpha_{p}^{2}<\left(k^{(0)}\right)^{2}\right\}, \\
e_{p}^{(M)}=\frac{\left|d_{p}^{(M)^{2}}\right| \beta_{p}^{(M)}}{\beta} \quad p \in D^{(M)}=\left\{p \mid \alpha_{p}^{2}<\left(k^{(M)}\right)^{2}\right\},
\end{gathered}
$$

which characterize the "outgoing energy fraction" propagating away from the structure upward and downward, respectively. Conservation of energy is now stated precisely as

$$
\sum_{p \in U^{(0)}} e_{p}^{(0)}+\sum_{p \in D^{(M)}} e_{p}^{(M)}=1,
$$

and we can use as a diagnostic of convergence the "energy defect"

$$
\delta=1-\sum_{p \in U^{(0)}} e_{p}^{(0)}-\sum_{p \in D^{(M)}} e_{p}^{(M)} .
$$

In Table II, we display results of this energy defect, $\delta$, as $N$, the number of terms retained in the Taylor series is increased. Clearly, the convergence is exponential (down to machine zero) as we would expect.

In the five-layer case, we chose

TABLE II. Energy defect $(\delta)$ versus number of Taylor series terms retained, cf. Eq. (25), in a simulation of scattering by a three-layer structure. Physical parameters are reported in Eq. (28) while the numerical parameters were $N_{x}=128$ and $N_{\max }=24$.

\begin{tabular}{lc}
\hline \hline$N$ & Energy defect $(\delta)$ \\
\hline 0 & 0.00547926 \\
2 & 0.00206438 \\
4 & $-2.27676 \times 10^{-5}$ \\
6 & $-1.52311 \times 10^{-7}$ \\
8 & $3.93408 \times 10^{-8}$ \\
10 & $-3.12122 \times 10^{-9}$ \\
12 & $1.76252 \times 10^{-10}$ \\
14 & $-7.7639 \times 10^{-12}$ \\
16 & $2.60902 \times 10^{-13}$ \\
18 & $-5.44009 \times 10^{-15}$ \\
20 & $-3.33067 \times 10^{-16}$ \\
22 & 0 \\
24 & 0 \\
\hline \hline
\end{tabular}


TABLE III. Energy defect $(\delta)$ versus number of Taylor series terms retained, cf. Eq. (25), in a simulation of scattering by a five-layer structure. Physical parameters are reported in Eq. (30) while the numerical parameters were $N_{x}=128$ and $N_{\max }=24$.

\begin{tabular}{lc}
\hline \hline$N$ & Energy defect $(\delta)$ \\
\hline 0 & 0.0197169 \\
2 & 0.0171099 \\
4 & -0.000155799 \\
6 & $-4.50847 \times 10^{-5}$ \\
8 & $3.0206 \times 10^{-6}$ \\
10 & $-8.05347 \times 10^{-8}$ \\
12 & $2.20133 \times 10^{-9}$ \\
14 & $-5.54635 \times 10^{-10}$ \\
16 & $7.60854 \times 10^{-11}$ \\
18 & $-5.36948 \times 10^{-12}$ \\
20 & $1.56319 \times 10^{-13}$ \\
22 & $8.21565 \times 10^{-15}$ \\
24 & $2.17604 \times 10^{-14}$ \\
\hline \hline
\end{tabular}

$$
\begin{aligned}
& \beta^{(m)}=(1.1,2.2,3.3,4.4,5.5), \\
& a^{(m)}=(1.5,0.5,-0.5,-1.5), \\
& g^{(m)}=(\cos (x), \sin (2 x), \cos (3 x), \sin (4 x)),
\end{aligned}
$$

and $\varepsilon=0.1$. Again, for numerical parameters, we selected $N_{x}=128$ and $N=24$. In Table III, we display results of this energy defect, $\delta$, as $N$, the number of terms retained in the Taylor series is increased. Again, we note exponential convergence (down to machine zero) as expected.

To conclude, we present results of some preliminary numerical simulations of a point-source disturbance within the lowest layer meant to be a very crude model of a subterranean earthquake which fits into our periodic framework. As we saw in Eq. $(2 \mathrm{~g})$, the incident radiation can be quite general and our point-source model is no exception provided that we consider a periodic family of point sources, which is quite natural given the periodic nature of our interfaces. With this specification, we recall that such a function can be

TABLE IV. Efficiency $e_{0}$ and relative error (compared to the highest accuracy solution) versus number of Taylor series terms retained, cf. Eq. (25), in a simulation of point-source scattering by a three-layer structure. Physical parameters are reported in Eq. (28) while the numerical parameters were $N_{x}=128$ and $N_{\max }=24$.

\begin{tabular}{lcc}
\hline \hline$N$ & $e_{0}^{N}$ & $e_{0}^{N}\left|e_{0}^{N}-e_{0}^{24}\right| /\left|e_{0}^{24}\right|$ \\
\hline 0 & $2.638809126959936 \times 10^{-5}$ & 0.352445 \\
2 & $4.317263372822617 \times 10^{-5}$ & 0.0594416 \\
4 & $4.063250064761511 \times 10^{-5}$ & 0.00289242 \\
6 & $4.075330989714637 \times 10^{-5}$ & $7.21934 \times 10^{-5}$ \\
8 & $4.075037414093768 \times 10^{-5}$ & $1.50906 \times 10^{-7}$ \\
10 & $4.075036372657268 \times 10^{-5}$ & $1.04659 \times 10^{-7}$ \\
12 & $4.075036820779545 \times 10^{-5}$ & $5.30827 \times 10^{-9}$ \\
14 & $4.075036798603324 \times 10^{-5}$ & $1.33696 \times 10^{-10}$ \\
16 & $4.075036799146814 \times 10^{-5}$ & $3.2559 \times 10^{-13}$ \\
18 & $4.075036799148951 \times 10^{-5}$ & $1.98713 \times 10^{-13}$ \\
20 & $4.075036799148102 \times 10^{-5}$ & $9.64466 \times 10^{-15}$ \\
22 & $4.075036799148142 \times 10^{-5}$ & $1.66287 \times 10^{-16}$ \\
24 & $4.075036799148141 \times 10^{-5}$ & 0 \\
\hline \hline
\end{tabular}

TABLE V. Efficiency $e_{-2}$ and relative error (compared to the highest accuracy solution) versus number of Taylor series terms retained, cf. Eq. (25), in a simulation of point-source scattering by a three-layer structure. Physical parameters are reported in Eq. (28) while the numerical parameters were $N_{x}=128$ and $N_{\max }=24$.

\begin{tabular}{lcc}
\hline \hline$N$ & $e_{-2}^{N}$ & $e_{-2}^{N}\left|e_{-2}^{N}-e_{-2}^{24}\right| /\left|e_{-2}^{24}\right|$ \\
\hline 0 & 0.0005213256160486521 & 0.243832 \\
2 & 0.0006943499382756026 & 0.00713487 \\
4 & 0.0006894970545861178 & $9.58966 \times 10^{-5}$ \\
6 & 0.0006894225965224039 & $1.21027 \times 10^{-5}$ \\
8 & 0.0006894312866295445 & $5.02008 \times 10^{-7}$ \\
10 & 0.0006894309333885411 & $1.03583 \times 10^{-8}$ \\
12 & 0.0006894309404349986 & $1.37632 \times 10^{-10}$ \\
14 & 0.0006894309405442724 & $2.08666 \times 10^{-11}$ \\
16 & 0.0006894309405292778 & $8.82703 \times 10^{-13}$ \\
18 & 0.0006894309405298982 & $1.71414 \times 10^{-14}$ \\
20 & 0.0006894309405298865 & $1.5726 \times 10^{-16}$ \\
22 & 0.0006894309405298864 & 0 \\
24 & 0.0006894309405298864 & 0 \\
\hline \hline
\end{tabular}

defined with the upward propagating, periodized free-space Green's function ${ }^{33}$

$$
G_{q p}(x, y)=-\frac{i}{4} \sum_{p=-\infty}^{\infty} e^{i \alpha p d} H_{0}^{(1)}\left(k \sqrt{(x-p d)^{2}+y^{2}}\right)
$$

where $H_{0}^{(1)}$ is the zeroth-order Hankel function of the first kind. If we desire a singularity (e.g., an epicenter) at $\left(x_{0}, y_{0}\right)$, then the point-source is given by

$$
w_{p s}(x, y)=G_{q p}\left(x-x_{0}, y-y_{0}\right) \text {. }
$$

For utilization in our recursions, it is more convenient to use the spectral representation ${ }^{33}$

$$
G_{q p}(x, y)=\frac{1}{2 i d} \sum_{p=-\infty}^{\infty} \frac{e^{i\left(\alpha_{p} x+\beta_{p}|y|\right)}}{\beta_{p}},
$$

and, again, $w_{p s}(x, y)=G_{q p}\left(x-x_{0}, y-y_{0}\right)$. Setting $w_{i}=w_{p s}$, $\left(x_{0}, y_{0}\right)=(d / 2,-20)$ and $\alpha=0$ (so that the point sources are periodic rather than quasiperiodic), we can now test the capabilities of our method in the three-layer configuration outlined above; cf. Eq. (28) with $\varepsilon=0.1$. In Tables IV and $\mathrm{V}$, we report computations of the scattering efficiencies $e_{0}$ and $e_{-2}$, respectively, in the upper layer as the perturbation order $N$ is increased. As we have seen in all of the simulations above, a rapid and stable convergence of the efficiency is displayed as the perturbation order is increased resulting in full double precision accuracy by $N=24$.

\section{ACKNOWLEDGMENTS}

D.P.N. gratefully acknowledges support from the National Science Foundation through Grant No. DMS0810958 and the Department of Energy under Award No. DE-SC0001549.

${ }^{1}$ P. G. Dinesen and J. S. Hesthaven, "Fast and accurate modeling of waveguide grating couplers," J. Opt. Soc. Am. A 17, 1565-1572 (2000). 
${ }^{2}$ P. G. Dinesen and J. S. Hesthaven, "Fast and accurate modeling of waveguide grating couplers. II. Three-dimensional vectorial case," J. Opt. Soc. Am. A 18, 2876-2885 (2001).

${ }^{3}$ L. C. Wilcox, P. G. Dinesen, and J. S. Hesthaven, "Fast and accurate boundary variation method for multilayered diffraction optics," J. Opt. Soc. Am. A 21, 757-769 (2004).

${ }^{4}$ M.-S. Min, T.-W. Lee, P. F. Fischer, and S. K. Gray, "Fourier spectral simulations and Gegenbauer reconstructions for electromagnetic waves in the presence of a metal nanoparticle,” J. Comput. Phys. 213, 730-747 (2006).

${ }^{5}$ L. Tsang, J. A. Kong, and R. T. Shin, Theory of Microwave Remote Sensing (Wiley-Interscience, New York, 1985), pp. 1-613.

${ }^{6} \mathrm{~J}$. Virieux and S. Operto, "An overview of full-waveform inversion in exploration geophysics," Geophysics 74, WCC1-WCC26 (2009).

${ }^{7}$ F. Bleibinhaus and S. Rondenay, "Effects of surface scattering in fullwaveform inversion," Geophysics 74, WCC69-WCC77 (2009).

${ }^{8}$ L. Geli, P. Bard, and B. Jullien, "The effect of topography on earthquake ground motion: A review and new results," Bull. Seismol. Soc. Am. 78, 42-63 (1988).

${ }^{9}$ F. J. Sanchez-Sesma, V. J. Palencia, and F. Luzon, "Estimation of local site effects during earthquakes: An overview," in From Seismic Source to Structural Response, edited by V. K. Gupta (Department of Civil Engineering, University of Southern California, 2004), pp. 44-70.

${ }^{10} \mathrm{P}$. Moczo, J. Robertsson, and L. Eisner, "The finite-difference time-domain method for modeling of seismic wave propagation," Adv. Geophys. 48, 421-516 (2007).

${ }^{11}$ R. G. Pratt, "Frequency-domain elastic wave modeling by finite differences: A tool for crosshole seismic imaging," Geophysics 55, 626-632 (1990).

${ }^{12}$ O. C. Zienkiewicz, The Finite Element Method in Engineering Science, 3rd ed. (McGraw-Hill, New York, 1977), pp. 1-787.

${ }^{13}$ K. Koketsu, H. Fujiwara, and Y. Ikegami, "Finite-element simulation of seismic ground motion with a voxel mesh," Pure Appl. Geophys. 161, 2183-2198 (2004).

${ }^{14}$ D. Komatitsch and J. Tromp, "Spectral-element simulations of global seismic wave propagation-I. Validation,” Geophys. J. Int. 149, 390-412 (2002).

${ }^{15}$ D. Komatitsc and J. Tromp, "Spectral-element simulations of global seismic wave propagation-II. Three-dimensional models, oceans, rotation and self-gravitation," Geophys. J. Int. 150, 303-318 (2002).

${ }^{16}$ F. Sanchez-Sesma, E. Perez-Rocha, and S. Chavez-Perez, "Diffraction of elastic waves by three-dimensional surface irregularities. Part II," Bull. Seismol. Soc. Am. 79, 101-112 (1989).

${ }^{17}$ M. Bouchon, "A review of the discrete wavenumber method," Pure Appl. Geophys. 160, 445-465 (2003).

${ }^{18}$ L. Greengard and V. Rokhlin, "A fast algorithm for particle simulations," J. Comput. Phys. 73, 325-348 (1987).

${ }^{19}$ O. P. Bruno and F. Reitich, "Solution of a boundary value problem for the Helmholtz equation via variation of the boundary into the complex domain," Proc. -R. Soc. Edinburgh, Sect. A 122, 317-340 (1992).

${ }^{20}$ O. P. Bruno and F. Reitich, "Numerical solution of diffraction problems: A method of variation of boundaries," J. Opt. Soc. Am. A 10, 1168-1175 (1993).
${ }^{21}$ O. P. Bruno and F. Reitich, "Numerical solution of diffraction problems: A method of variation of boundaries. II. Finitely conducting gratings, Padé approximants, and singularities," J. Opt. Soc. Am. A 10, 2307-2316 (1993).

${ }^{22}$ O. P. Bruno and F. Reitich, "Numerical solution of diffraction problems: A method of variation of boundaries. III. Doubly periodic gratings," J. Opt. Soc. Am. A 10, 2551-2562 (1993).

${ }^{23}$ D. M. Milder, "An improved formalism for rough-surface scattering of acoustic and electromagnetic waves," in Proceedings of the SPIE-The International Society for Optical Engineering, San Diego, Bellingham, WA (1991), pp. 213-221.

${ }^{24}$ D. M. Milder, "An improved formalism for wave scattering from rough surfaces," J. Acoust. Soc. Am. 89, 529-541 (1991).

${ }^{25}$ D. M. Milder and H. T. Sharp, "Efficient computation of rough surface scattering," in Mathematical and Numerical Aspects of Wave Propagation Phenomena (SIAM, Strasbourg, Philadelphia, PA, 1991), pp. 314322.

${ }^{26}$ D. M. Milder and H. T. Sharp, "An improved formalism for rough-surface scattering. II: Numerical trials in three dimensions," J. Acoust. Soc. Am. 91, 2620-2626 (1992).

${ }^{27}$ D. M. Milder, "Role of the admittance operator in rough-surface scattering," J. Acoust. Soc. Am. 100, 759-768 (1996).

${ }^{28}$ D. M. Milder, "An improved formalism for electromagnetic scattering from a perfectly conducting rough surface," Radio Sci. 31, 1369-1376 (1996).

${ }^{29}$ D. P. Nicholls and F. Reitich, "Stability of high-order perturbative methods for the computation of Dirichlet-Neumann operators," J. Comput. Phys. 170, 276-298 (2001).

${ }^{30}$ D. P. Nicholls and F. Reitich, "Shape deformations in rough-surface scattering: Cancellations, conditioning, and convergence," J. Opt. Soc. Am. A 21, 590-605 (2004).

${ }^{31}$ D. P. Nicholls and F. Reitich, "Shape deformations in rough-surface scattering: Improved algorithms," J. Opt. Soc. Am. A 21, 606-621 (2004).

${ }^{32}$ D. P. Nicholls and F. Reitich, "Boundary perturbation methods for highfrequency acoustic scattering: Shallow periodic gratings," J. Acoust. Soc. Am. 123, 2531-2541 (2008).

${ }^{33}$ R. Petit, Electromagnetic Theory of Gratings (Springer-Verlag, Berlin, 1980), Chap. 15 , p. 284.

${ }^{34} \mathrm{G}$. Müller, "The reflectivity method: A tutorial," J. Geophys. 50, 153-174 (1985).

${ }^{35}$ L. C. Evans, Partial Differential Equations (American Mathematical Society, Providence, 1998), Chap. 18, p. 662.

${ }^{36}$ D. Gottlieb and S. A. Orszag, "Numerical analysis of spectral methods: Theory and applications," in Proceedings of the CBMS-NSF Regional Conference Series in Applied Mathematics, Society for Industrial and Applied Mathematics, Philadelphia (1977), Chap. 5, p. 172.

${ }^{37}$ G. A. Baker, Jr. and P. Graves-Morris, Pade Approximants and Numerical Methods, 2nd ed. (Cambridge University Press, Cambridge, 1996), Chap. 14 , p. 746 . 